\title{
THE SOLVENCY MARGIN \\ IN NON-LIFE INSURANCE COMPANIES
}

\author{
G. W. De Wit and W. M. Kastelijn
}

\section{InTRODUCTION}

Much attention has been paid in the last ten years to the necessary solvency margin especially as influenced by risk theory considerations. In these calculations, by which the solvency margin is determined in such a way that the probability of ruin remains under a specified norm, such factors as type of insurance, size of portfolio, reinsurance etc. play an essential role.

In these calculations, based on pure risk theory, there is thus no attention paid to risks in the investment field, in the area of costs, etc. Yet one must not forget that the solvency margin must also act as a buffer against these uncertainties, in other words the solvency margin must be large enough to cover all risks to which the concern is liable, within stipulated limits of certainty.

One drawback of a pure risk theoretical approach, as interpreted above, is that the bases of the risk theory are often difficult to translate into practical terms, and furthermore will differ from company to company. The latter is also the case with the size of portfolio, reinsurance, etc. However, if this could all be translated into practical terms in the right way, the results would upset relations between competitors, so that it seems natural to make the required solvency margin the same for all companies.

Seen in this light Campagne's approach is of great importance-already in 1948 he realized that a scientific basis for the size of the solvency margin was necessary ${ }^{1}$. He confined himself to the life insurance industry, and based his calculations on the idea that the total results of the companies working in a country should be such that, ceteris paribus, the chance of bankruptcy remains below a stipulated norm.

These same ideas are worked out by Campagne for several countries and for the life and non-life insurance industries in a report entitled: "Standard minimum de solvabilité, applicable aux entreprises d'assurances" ${ }^{2}$. In par. 2 of Chapter I (Modèle statistique du portefeuille de l'assureur-dommages) is laid down what information one needs for a risk theoretical approach, in the same way as mentioned above. The O.E.C.D. also finds that this information is in general not available and therefore in practice chooses a simplified model

1 C. Campagne: Contribution to the method of calculating the stabilization reserve in life assurance business, Gedenkboek Verzekeringskamer 1923-1948, 1948, $33^{8} \mathrm{ff}$.

2 O.E.C.D., $11 \cdot 3 \cdot 1961$. 
(see numbers 65 up to and including 73 under the heading: simplification du modèle), from which follows an indication of the danger zone for the individual non-life insurance company as a whole.

The observations on which this report is based are mostly taken from the fifties. Since then, however, the ratios, such as those between claims and expenses, and also the results of the companies have changed drastically.

There is thus every reason to update the results of this research. Particularly so, because the rules concerning the size of the solvency margin contained in the European directives are based on the conclusions of this report.

This updating for the Dutch non-life insurance is worked out below, beginning with the reconstruction of the O.E.C.D. calculations applying to the Netherlands.

\section{O.E.C.D. REPORT}

From the conceptions dealt with in this report we shall discuss two:

- the expense ratio, defined as the expenses and commission after deduction of the commission received from reinsurers, expressed as a percentage of the net received premium.

- the claims ratio, defined as the claims paid for own account expressed as a percentage of the net received premium.

The information analysed referred to the period 1952-1957.

For the Netherlands information was included from 10 companies, which produced in total 53 figures.

The 53 claims ratios calculated in this manner and arranged according to size show the following picture:



Fig. 1 
The average expense ratio worked out at 53 and the average claims ratio at 43. The distribution of the claims ratios according to size was approached by means of a beta distribution.

$$
\begin{aligned}
f(x ; p, q) & =\frac{x^{p-1}(1-x)^{q-1}}{B(p, q)} & & \text { for } 0<x<1 \\
& =0 & & \text { for } x \leqslant \text { o or } x \geqslant 1,
\end{aligned}
$$

with $B(p, q)=\int_{0}^{1} t^{p-1}(1-t)^{q-1} d t$

$p$ and $q$ are the parameters of the distribution, $x$ the claims ratio. From the definition of the beta distribution it appears that the chance of claims ratios greater than $100 \%$ is nil. In practice it appeared that claims ratios greater than $66 \%$ did not occur.

The mean of the beta distribution is:

and the variance:

$$
\mu=\frac{p}{p+q}
$$

$$
\sigma^{2}=\frac{p q}{(p+q)^{2}(p+q+1)}
$$

As stated above, the value of the mean for the 10 Dutch companies was found to be 0.43 . The value of the standard deviation was not explicitly stated, but this must have been about 0.089 . The corresponding values of $p$ and $q$ then become:

$$
\begin{aligned}
& p=12.9 \\
& q=16.9
\end{aligned}
$$

Making use of the distribution laid down above, the claims ratio which has a probability of ruin of $0.3 \%$, comes out at 78 . That means that, if one can finance a claims ratio of 78 with the total security, the chance of bankruptcy is only 3 in 10,000 .

The calculation of the solvency margin is then:

$\begin{array}{lr}\text { net retained premium } & 100 \\ \text { expense ratio } & 53 \\ \text { for claims payments remains } & 47 \\ \text { maximum claims ratio } & 78 \\ \text { solvency margin } & 31\end{array}$

3. UPDATING

The calculating method shown in par. 2 is updated with figures for the years 
1976, 1977 and 1978. In total the information from 71 companies was available, from which 213 figures emerged. It may be expected that the 71 companies give a representative picture of the Dutch non-life insurance industry.

The collected information only applies to the industry in the Netherlands. Considering the availability of the material it was not possible to define the expense ratio and the claims ratio in exactly the same way as in the O.E.C.D. report. The following definitions had to suffice:

- expense ratio: the expenses and commission, before deduction of the commission received from reinsurers, expressed as a percentage of the gross earned premium.

- claims ratio: the gross incurred claims expressed as a percentage of the gross earned premium.
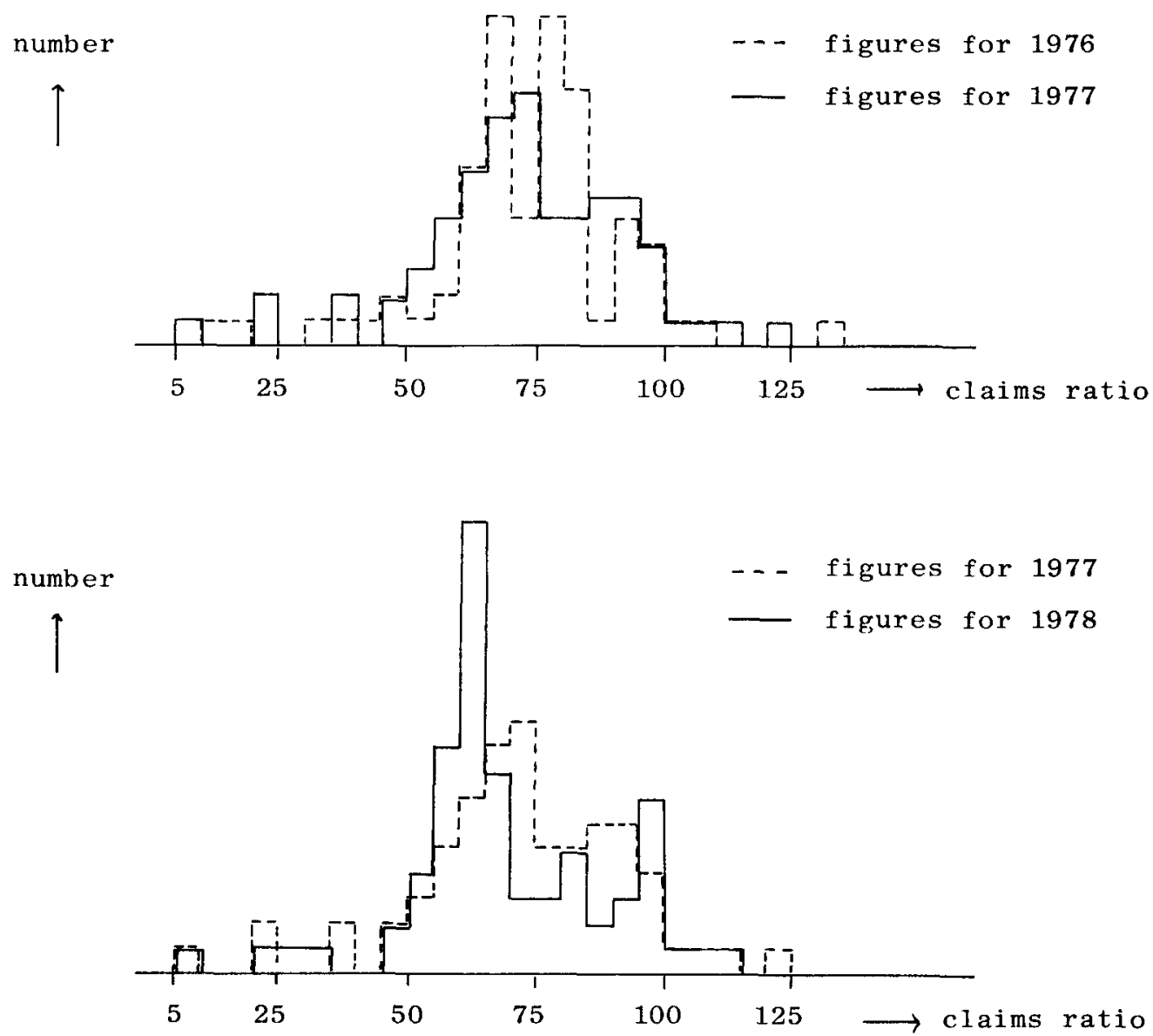

Fig. 2. 
The claims ratios thus defined resulted in the following histograms for the 71 Dutch companies.

The means for these years are as follows:

TABLE 1

\begin{tabular}{ccc}
\hline \multirow{2}{*}{ Year } & \multicolumn{2}{c}{ Claims ratio } \\
& mean & standard deviation \\
\hline 1976 & 734 & 188 \\
1977 & 725 & 200 \\
1978 & 691 & 193 \\
\hline $1976-1978$ & 717 & 194 \\
\hline
\end{tabular}

A few conclusions:

- the average claims ratio is considerably higher than that observed in the O.E.C.D. report for the period 1952-1957. The standard deviation also shows a significant increase. The following diagram gives the mean, the mean plus the standard deviation and the mean minus the standard deviation.

TABLE 2

\begin{tabular}{cccc}
\hline & Mean & Mean - standard deviation & Mean + standard deviation \\
\hdashline$-1952-1957$ & 430 & 341 & 519 \\
$1976-1978$ & 717 & $5^{2} 3$ & 911 \\
\hline
\end{tabular}

- the figures for the separate years show a slight drop. This drop can be the result of a more favourable claims experience or of a more adequate premium volume.

\section{DISTRIBUTION OF CLAIMS RATIOS}

For the analysis of the above-mentioned claims ratios basically the same method has been used as in the O.E.C.D. report, i.e. the application of a beta distribution. In this case, however, a correction must be made.

The histograms show that claims ratios greater than $100 \%$ occur rather frequently. Therefore, it would not be correct to approach the distribution by means of a beta distribution, which is defined between o and 100. For the determination of a higher upper limit for the beta distribution the upper limit at which the theoretical distribution gives a good approximation for the distribution observed was studied (see diagram). It was found that the upper 
limit must be approximately twice the mean, which is the same ratio as found in the O.E.C.D. report. Thus a distribution between o and 150 was chosen.

The approximation by means of the beta distribution consists of two steps:

1. transformation of the claims ratios by dividing by 1.5 , so that the transformed ratio indeed ranges from o to 100 ,

2. calculation of the values of $p$ and $q$ according to the formulas of paragraph 2 , so that the beta distribution is conclusively determined.

The following table summarizes the calculations:

TABLE 3

\begin{tabular}{|c|c|c|c|c|c|c|}
\hline \multirow[b]{2}{*}{ Year } & \multicolumn{2}{|c|}{ Claims ratio } & \multicolumn{2}{|c|}{ Claims ratio divided by 1.5} & \multirow[b]{2}{*}{$p$} & \multirow[b]{2}{*}{$q$} \\
\hline & mean & stand. dev. & mean & stand. dev. & & \\
\hline 1976 & 73.4 & 18.8 & 48.9 & 12.5 & $7 \cdot 30$ & 7.63 \\
\hline 1977 & 72.5 & 20.0 & 48.3 & 13.4 & 6.28 & 6.72 \\
\hline 1978 & 69.1 & 19.3 & 46.1 & 12.8 & $6.4^{8}$ & $7 \cdot 5^{8}$ \\
\hline $1976-1978$ & 71.7 & 19.4 & 47.8 & 12.9 & 6.68 & $7 \cdot 30$ \\
\hline
\end{tabular}

For the years 1976-1978 the following diagram shows the calculation by means of the beta distribution:

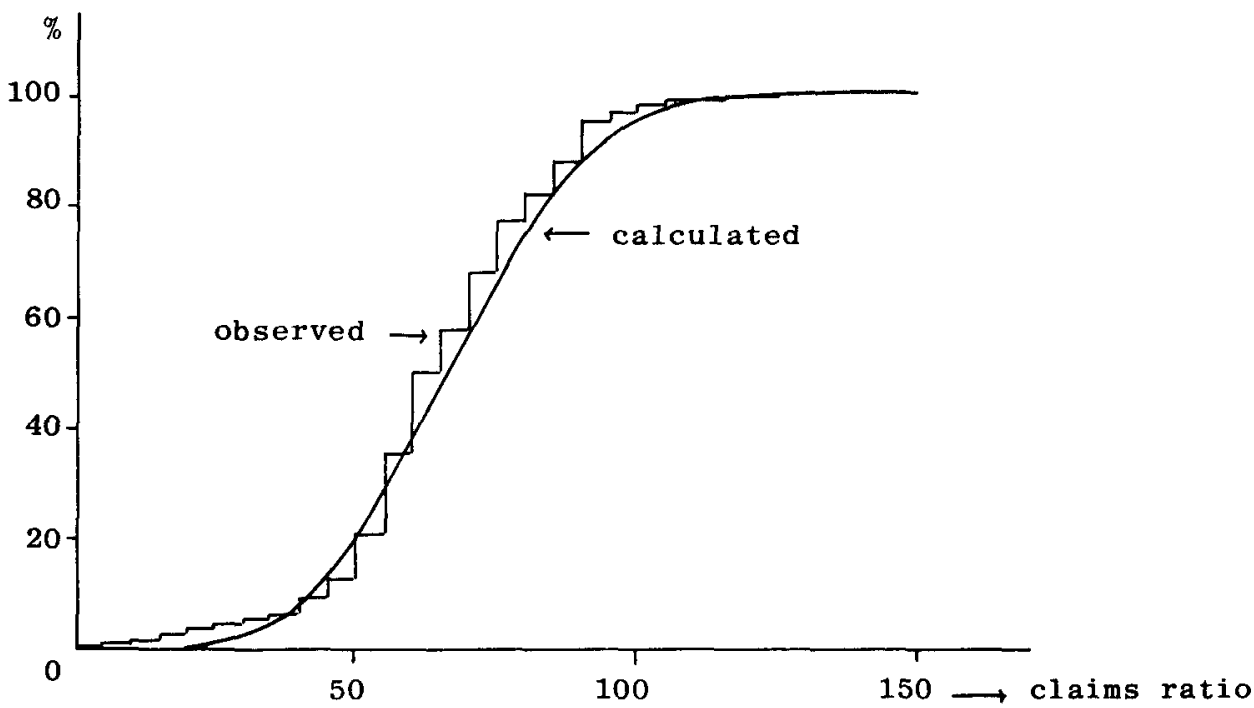

Fig. 3. Cumulative distribution of the claims ratios 1976-1978. 


\section{EXPENSE RATIO}

The average expense ratio for the Dutch companies studied is considerably lower for the period $1976-1978$ than at the time of the O.E.C.D. report.

Moreover, a further downward trend can be observed:

TABLE 4

\begin{tabular}{cc}
\hline Year & Expense ratio \\
\hline 1976 & 304 \\
1977 & 301 \\
1978 & 299 \\
\hline $1976-1978$ & 302 \\
\hline
\end{tabular}

\section{RESULTS}

Analogous to the analysis in the O.E.C.D. report, the period 1976-1978 shows the following results, calculated for various probabilities of ruin, i.e. chances of bankruptcy, and based on an average expense ratio of 30 :

TABLE 5

\begin{tabular}{|c|c|c|c|}
\hline & \multicolumn{3}{|c|}{ Probability of ruin } \\
\hline & $1 \%$ & $1 \% 0$ & $03 \% 0$ \\
\hline earned premium & 100 & 100 & 100 \\
\hline expense ratio & 30 & 30 & 30 \\
\hline for claims payments remains & 70 & 70 & 70 \\
\hline maxımum claims ratıo & 115 & 126 & $13^{\circ}$ \\
\hline solvency margin & 45 & 56 & 60 \\
\hline
\end{tabular}

A few remarks:

- when the same, very strict, solvency requirement is applied as in the O.E.C.D. report $(0.3 \%$ ) , the necessary solvency margin is $60 \%$, instead of the $31 \%$ calculated at the time.

- the level of the solvency margin is not only determined by the claims and expense ratio, but also, and to an even more significant degree, by the standard deviation of the figures. In the following diagram, which gives the solvency margin for three values of the standard deviation, this dependence can clearly be observed. 


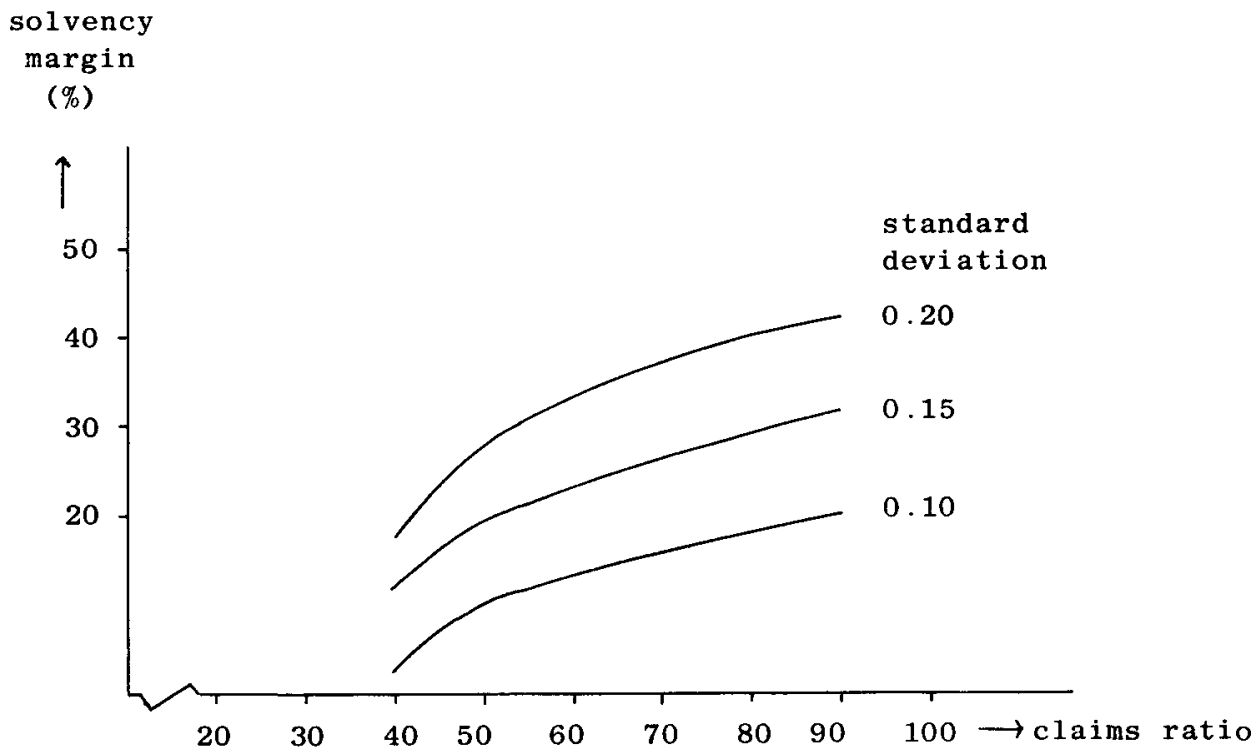

Fig. 4 .

\section{OTHER DISTRIBUTION OF CLAIMS RATIOS}

The above figures are all based on a beta distribution, analogous to the methods applied for the O.E.C.D. report. In paragraph 4 the objection to the upper limit has already been mentioned. Empirically, this was set at 150 .

A distribution which does not have this disadvantage is the Weibull distribution ${ }^{3}$ :

$$
\begin{aligned}
& g(x ; a, b)=\frac{b}{a}\left(\frac{x}{a}\right)^{b-1} \exp \left[-\left(\frac{x}{a}\right)^{b}\right] \quad \text { for } x \geqslant 0 \\
& =0 \quad \text { for } x<0
\end{aligned}
$$

Estimation of $a$ and $b$ with the principle of maximum likelihood gives:

$$
\begin{aligned}
& a=78.6 \\
& b=4.12
\end{aligned}
$$

${ }_{3}$ The application of the Weibull distribution was brought to our attention by $\mathrm{P}$. ter Berg, staff member of the Bureau voor Statistiek en Onderzoek of the Verbond van Verzekeraars in Nederland. 
The following table shows a close similarity of these two distributions:

TABLE 6

\begin{tabular}{|c|c|c|c|}
\hline & \multicolumn{3}{|c|}{ Probability of ruin } \\
\hline & $1 \%$ & $1 \% 0$ & $0.3 \% 0$ \\
\hline \multirow{2}{*}{ maximum claims ratio $\left\{\begin{array}{l}\text { Weibull } \\
\text { beta }\end{array}\right.$} & 113.9 & 125.6 & 130.6 \\
\hline & 115.4 & 126.2 & 130.2 \\
\hline
\end{tabular}

There is a definite similarity between the maximum claims ratios on the basis of a beta distribution and on the basis of a Weibull distribution.

\section{FINAL REMARK}

A large number of European countries have been analysed in the O.E.C.D. report. In the above this has been updated for the Netherlands. It would be advisable to do the same for other European countries. 